\title{
LiNiO Gate Dielectric with Tri-Gate Structure for High Performance E-mode GaN transistors
}

\author{
Taifang Wang, Mohammad Samizadeh Nikoo, Luca Nela, and Elison Matioli \\ Ecole polytechnique federale de Lausanne (EPFL), Lausanne, Switzerland. \\ email:taifang.wang@epfl.ch, elison.matioli@epfl.ch
}

\begin{abstract}
In this work, a Tri-Gate AlGaN/GaN metal-oxidesemiconductor high electron mobility transistor (MOSHEMT) with lithium nickel oxide $(\mathrm{LiNiO})$ gate dielectric is demonstrated for enhancement-mode (e-mode) operation. The high-quality of pulse-laser-deposited (PLD) LiNiO resulted in e-mode devices without the need for special epitaxial layers, barrier recess, or regrowth. The $\mathrm{LiNiO}$ Tri-Gate devices presented a positive Vth, low Ron, large maximum on-current (Ion, max), and high breakdown voltage (Vbr) simultaneously. LiNiO also yielded excellent negative bias temperature instability (NBTI) performance, small hysteresis, and small frequency dispersion.

Index Terms - GaN, HEMT, Enhancement-mode, NiO, LiNiO, trigate, Normally-off.
\end{abstract}

\section{INTRODUCTION}

$\mathrm{P}$

-GaN gates are currently the technology mostly adopted commercially for e-mode GaN-on-Si high electron mobility transistors (HEMTs) due to its high reliability and stability[1]. However, to successfully reach high performance, this technology requires a thin-barrier epitaxy, selective etching $\mathrm{p}-\mathrm{GaN}$ layer or re-growth of the barrier layer to meet the requirements of low $R_{\text {on }}$ and positive $V_{\text {th }}$. An alternative approach is to use p-type oxides as a flexible, and activation free replacement for $\mathrm{p}-\mathrm{GaN}$, which offers simple deposition and patterning methods. In this work, a Tri-Gate AlGaN/GaN metal-oxide-semiconductor high electron mobility transistor (MOSHEMT) with lithium nickel oxide $(\mathrm{LiNiO})$ gate dielectric is demonstrated for enhancement-mode (e-mode) operation. The high-quality of pulse-laser-deposited (PLD) $\mathrm{LiNiO}$ resulted in e-mode devices without the need for special epitaxial layers, barrier recess, or regrowth. The LiNiO TriGate devices presented a positive $\mathrm{V}_{\text {th }}$, low $\mathrm{R}_{\text {on }}$, large maximum on-current $\left(\mathrm{I}_{\mathrm{on}, \max }\right)$, and high breakdown voltage $\left(\mathrm{V}_{\mathrm{br}}\right)$ simultaneously. $\mathrm{LiNiO}$ also yielded excellent negative bias temperature instability (NBTI) performance, small hysteresis, and small frequency dispersion.

\section{DeVice StRUCtURE AND FABrication}

The device schematic is illustrated in Fig. 1 (a). The crosssection TEM image of fin region is shown in Fig. 1 (b). To understand the band offset between LiNiO and AlGaN XPS analysis and UV-Vis (Fig. 1 (c)) were performed. In this work, different Li contents were investigated, revealing that the higher Li-content $\mathrm{LiNiO}$ films presented larger valence band offset
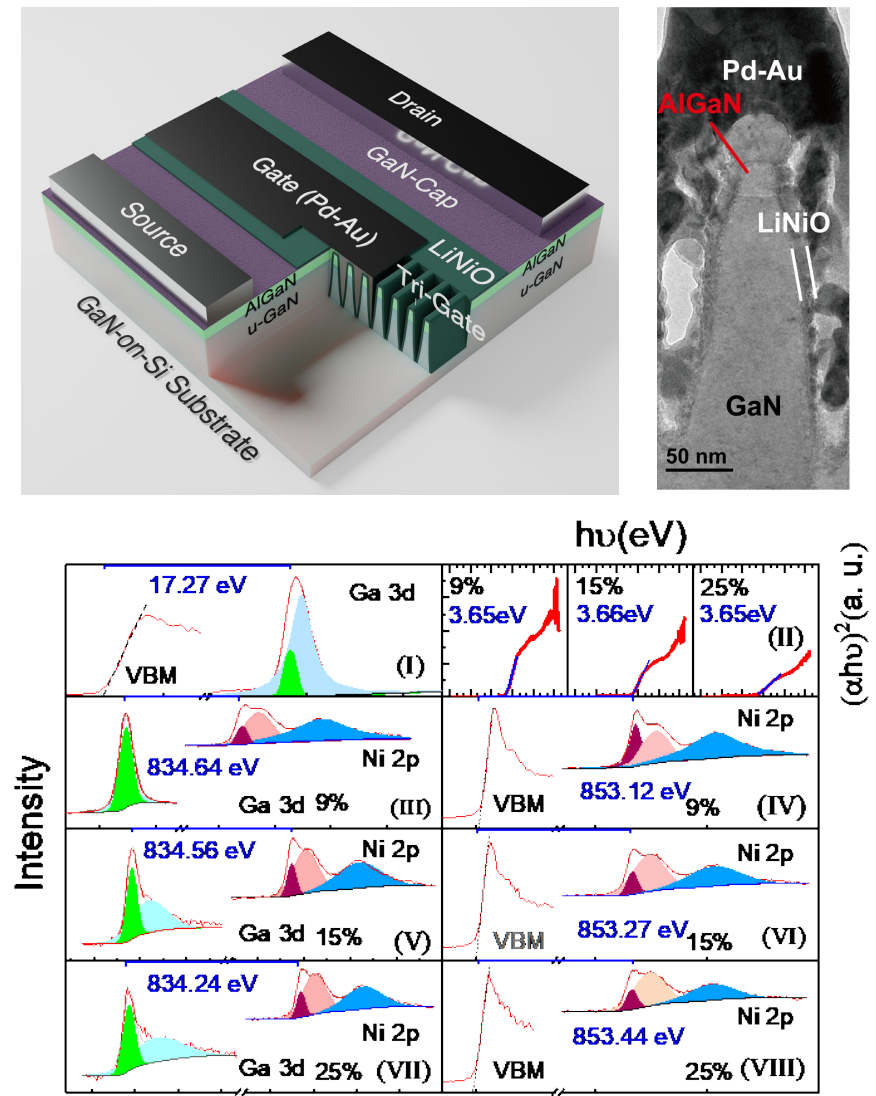

(c)

Binding energy (eV)

Figure 1. (a) Schematic of the device and cross-sectional view of tri-gate MOSHEMT structure with $\mathrm{LiNiO}$ gate dielectric. (b) cross-sectional transmission electron microscope (TEM) image of the LiNiO film on AlGaN/GaN tri-gate structure. (c) X-ray photoelectron spectroscopy (XPS): (I) valence band and $\mathrm{Ga} 3 \mathrm{~d}$ core level spectra for $\mathrm{AlGaN} / \mathrm{GaN}$. (III)(V)(VII) core level spectra of Ga $3 \mathrm{~d}$ and $\mathrm{Ni} 2 \mathrm{p}$ from $~ 3 \mathrm{~nm}$ thick $9 \%, 15 \%, 25 \% \mathrm{Li}$ doped $\mathrm{NiO}$ on AlGaN/GaN. (IV)(VI)(VIII) Ni $2 p$ core level spectra and valence band for $\sim 60 \mathrm{~nm}$ thick $9 \%, 15 \%, 25 \% \mathrm{Li}$ doped $\mathrm{NiO}$ on $\mathrm{AlGaN} / \mathrm{GaN}$. (II) absorption $\left((\alpha h v)^{2}\right)$ versus $h v$ optical transmission spectra plot from ultraviolet-visible spectroscopy (UV-Vis) for different $\mathrm{Li}$ doping concentration $\mathrm{LiNiO}$ film on fused silica, band gap values are extracted from these absorption spectra.

$\left(\Delta \mathrm{E}_{\mathrm{V}}\right)$ (Fig. 2 (a)), which is not ideal for e-mode operation. This was confirmed from gated-hall measurements on HEMT structures covered by LiNiO films and Pd gate, which showed a reduction in sheet resistance as the Li-content increased (Fig. 2 (b)), based on this, $9 \% \mathrm{Li}$-content film was used in the device fabrication. By depositing LiNiO layers over Tri-Gate

Union's Horizon 2020 research and innovation programme and Austria, Belgium, Germany, Italy, Norway, Slovakia, Spain, Sweden, Switzerland. grant agreement No 826392. The JU receives support from the European 


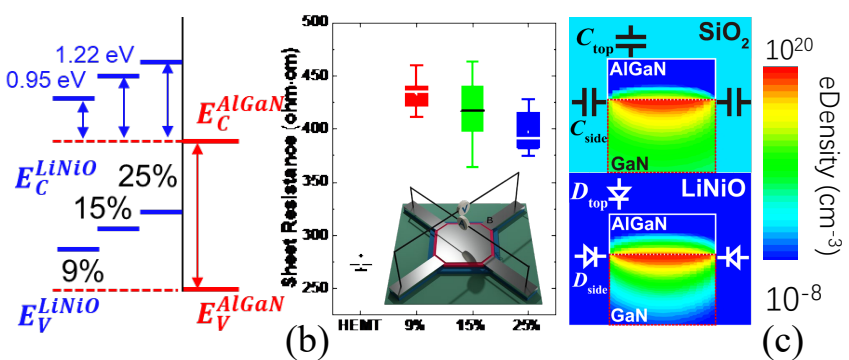

Figure 2. (a) Band alignment between $\mathrm{LiNiO}$ and $\mathrm{AlGaN}$ determined from XPS and UV-Vis measurements, for Li content of $9 \%, 15 \%$ and $25 \%$. (b) Measured sheet resistance on hall structures patterned on an $\mathrm{AlGaN} / \mathrm{GaN}$ HEMT structure (black), and three HEMT structures covered with $\mathrm{LiNiO}$ with $\mathrm{Li}$ concentrations of $9 \%$ (red), $15 \%$ (green) and $25 \%$ (blue) and $\mathrm{Pd} / \mathrm{Au}$ gate. Inset: schematic of gated hall structure. (c) Simulated electron density distribution in gate width direction of a single fin for top: $\mathrm{LiNiO}$ and bottom: $\mathrm{SiO}_{2} .25 \mathrm{~nm}$ thick $\mathrm{Al}_{0.25} \mathrm{Ga}_{0.75} \mathrm{~N}, 30 \mathrm{~nm}$-wide $200 \mathrm{~nm}$-tall fins, and $40 \mathrm{~nm}$ thick oxide were used for both structure, $\mathrm{V}_{\mathrm{G}}=0 \mathrm{~V}, 5.5 \mathrm{eV}$ Schottky gate and ohmic gate were used for $\mathrm{SiO}_{2}$ and $\mathrm{LiNiO}$ respectively.

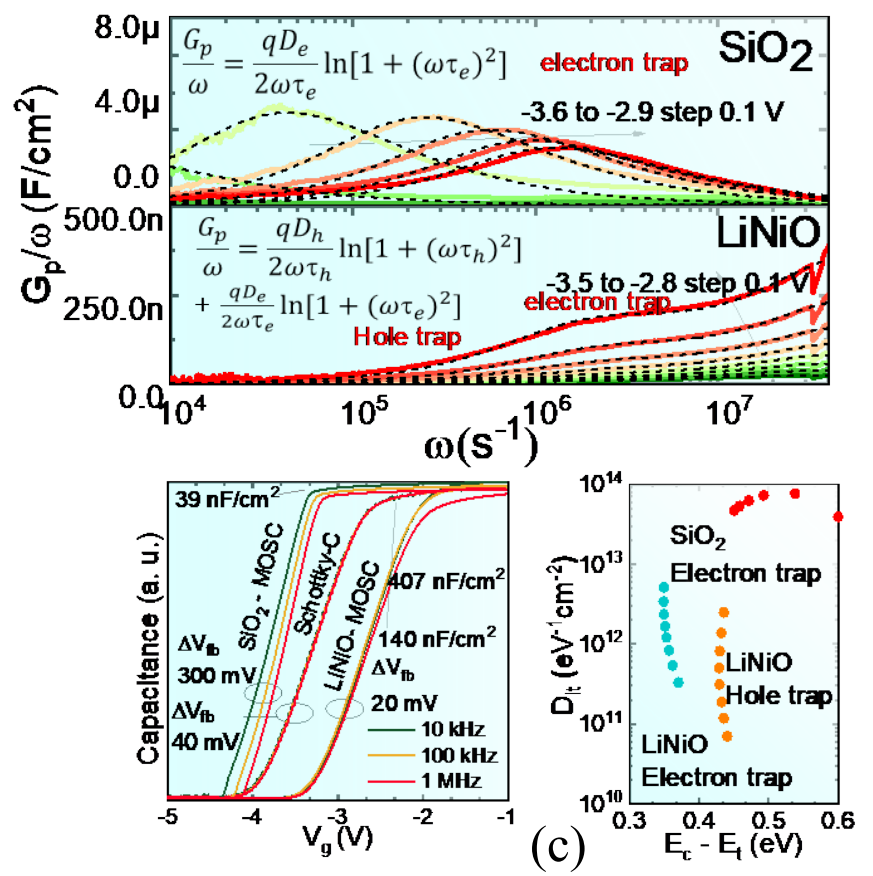

Figure 3. (a) Measured curves and fitting curves of $G_{p} / \omega$ vs $\omega$ at different bias voltage for $\mathrm{SiO}_{2} / \mathrm{AlGaN} / \mathrm{GaN}$ (top) and $\mathrm{LiNiO} / \mathrm{AlGaN}$ (bottom). A double trap equation was used for $\mathrm{LiNiO}$ MOSC and both are better fitted by continues trap level equation. (b) Multi-frequency $(10 \mathrm{k} \mathrm{Hz}, 100 \mathrm{kHz}$, and $1 \mathrm{MHz})$ capacitance versus gate voltage $(\mathrm{C}-\mathrm{V})$ characteristics of $\mathrm{SiO}_{2}$ MOSHEMT capacitor, $\mathrm{LiNiO}$ MOSHEMT capacitor, and Ni gate Schottky HEMT capacitor. First capacitor plateau value of three measurements are scaled to similar level for easier $\mathrm{V}_{\mathrm{fb}}$ shift comparison. $\mathrm{LiNiO}$ device shows nearly no dispersion at the rising edge of flatband voltage, while $\mathrm{SiO}_{2}$ device presents a much larger dispersion among different frequency. (c) Trap state density as a function of energy level for both device, corresponding parameters $\left(v_{\text {th }}, \sigma_{\mathrm{n},}, \sigma_{\mathrm{p}}, \mathrm{N}_{\mathrm{c}}, \mathrm{N}_{\mathrm{v}}\right)$ were used for hole and electron trap states in Shockley-Read-Hall statistics.

structures, the channel could be depleted from three sides of the Tri-Gate structures (Fig. 2 (c)).

\section{RESULTS AND DISCUSSION}

The interface properties of $\mathrm{LiNiO} / \mathrm{AlGaN}$ were studied. As shown in Fig. 3 (b), compared with $\mathrm{SiO}_{2}$ metal-oxidesemiconductor capacitor (MOSC) and Ni gate Schottky diode,
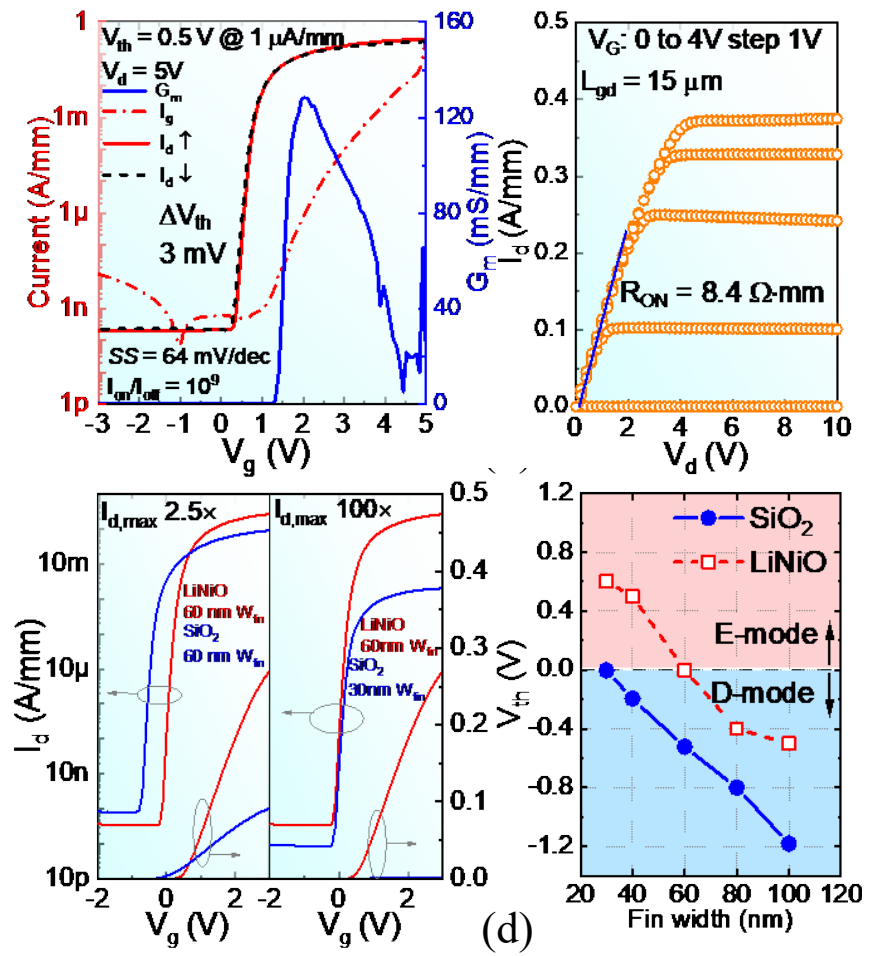

Figure 4. (a) Transfer characteristics of 40nm fin width Tri-Gate MOSHEMT with $9 \%$ Li-content $\mathrm{LiNiO}$ gate dielectric, measured at $\mathrm{V}_{\mathrm{d}}=5 \mathrm{~V}$, a $0.5 \mathrm{~V}$ (defined at $1 \mu \mathrm{A} / \mathrm{mm}$ ) $\mathrm{V}_{\text {th }}$ was achieved. To characterize hysteresis of device, a $10 \mathrm{mV}$ step and long integration time was used in Keysight B1505A to have a good voltage resolution and hash stress condition. (b) Output characteristic of LiNiO Tri-Gate MOSHEMT.A $\mathrm{R}_{\text {on }}$ of $8.4 \Omega \cdot \mathrm{mm}$ is from the linear fitting of $I_{d}$ at $\mathrm{Vg}=4 \mathrm{~V}, \mathrm{~V}_{\mathrm{d}}=1 \mathrm{~V}$. (c) Transfer characteristic comparison between $\mathrm{SiO}_{2}$ and $9 \% \mathrm{LiNiO}$ device under same fin width (left) or same $\mathrm{V}_{\text {th }}$ (right). Under same fin width, $V_{\text {th }}$ shift toward positive, and on-current also increase due to hole injection mode operation. (d) $V_{\text {th }}$ (defined at $1 \mu \mathrm{A} / \mathrm{mm}$ ) as a function of fin width and gate dielectric. The requirement of fin width to achieve e-mode operation greatly increase from $30 \mathrm{~nm}$ to $60 \mathrm{~nm}$ due to the junction depletion from the three side of fins.

LiNiO resulted in a small frequency dispersion in the $\mathrm{C}-\mathrm{V}$ curves, which is similar to the Schottky diode and much smaller than that of $\mathrm{SiO}_{2}$ dielectric (deposited by thermal ALD at $300^{\circ} \mathrm{C}$ ). To quantitatively study the interface trap states of LiNiO, a model comprised of parallel-connected $\mathrm{C}_{\mathrm{p}}$ and $\mathrm{G}_{\mathrm{p}}$ in series with a $\mathrm{C}_{\text {barrier }}+$ oxide was deployed with frequency dependent impedance measurement. In $\mathrm{LiNiO}$ MOSC, two peaks were observed (Fig. 3 (a)), the slower one is likely due to the hole traps at the $\mathrm{LiNiO} / \mathrm{AlGaN}$ interface, and the faster one is related to trap states the $\mathrm{AlGaN} / \mathrm{GaN}$ interface, which is also shown in the Schottky diode. The low density and fast emission time of trap states guarantee a high stability operation (Fig. 3(c)), Apart from the electron trap state (also presents in Ni/AlGaN/GaN Schottky capacitor), an additional trap state is shown, which results from the hole trap states at $\mathrm{LiNiO} / \mathrm{AlGaN}$ interface. By integrating of LiNiO with Tri-Gates, a $\mathrm{V}_{\text {th }}$ of $0.5 \mathrm{~V}$ (defined at $1 \mu \mathrm{A} / \mathrm{mm}$ ) was achieved with $40 \mathrm{~nm}$ fin width, with a subthreshold swing $(S S)$ of $64 \mathrm{mV} / \mathrm{dec}$, on/off ratio of $10^{9}$, peak transconductance $\left(\mathrm{G}_{\mathrm{m}}\right)$ of $130 \mathrm{mS} / \mathrm{mm}$ and small $\mathrm{V}_{\text {th }}$ hysteresis of $3 \mathrm{mV}$ (Fig. 4 (a)). $\mathrm{I}_{\text {on, } \max }$ of $380 \mathrm{~mA} / \mathrm{mm}$, and $\mathrm{R}_{\text {on }}$ of 8.4 $\Omega \cdot \mathrm{mm}$ were also observed (Fig. 4 (b)). Compared with Tri-Gate structures with $\mathrm{SiO}_{2}$, devices using $\mathrm{LiNiO}$ showed a $0.7 \mathrm{~V}$ positive shift in $\mathrm{V}_{\text {th }}$ and greatly improved the on-current for the same fin width (Fig. 4 (c) and (d)).

(C) 2021 IEEE. Personal use of this material is permitted. Permission from IEEE must be obtained for all other uses, in any current or future media, including reprinting/republishing this material for advertising or promotional purposes, creating new collective works, for resale or redistribution to servers or lists, or reuse of any copyrighted component of this work in other works. 

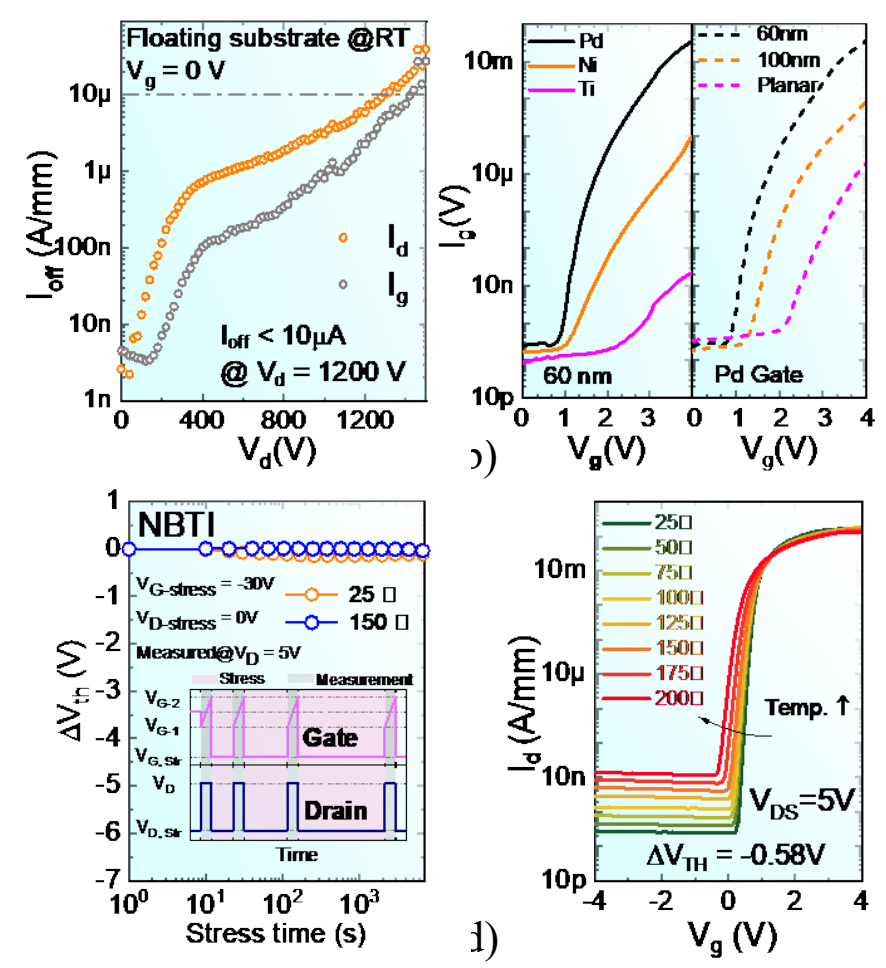

Figure 5. (a) Three terminal breakdown characteristics of devices at $\mathrm{V}_{\mathrm{g}}=0 \mathrm{~V}$ (floating substrate). Due to the usage of ohmic gate off-state leakage is higher than normal MOSHEMTs, however, the device still can hold a voltage over $1500 \mathrm{~V}$ without hard breakdown $\left(15 \mu \mathrm{m} \mathrm{L}_{\mathrm{gd}}\right)$. Engineering on gate leakage could further increase the soft breakdown voltage. (b) Gate leakage characteristic of devices with different gate metal (left) on $60 \mathrm{~nm}$ fin, and with different fin width (right) with Pd gate. (c) Threshold voltage shifts of $\mathrm{LiNiO}$ gate dielectric TriGate MOSHEMT during the gate bias stress of $\mathrm{V}_{\mathrm{g}}=-30 \mathrm{~V}$ at $25^{\circ} \mathrm{C}$ and $150^{\circ} \mathrm{C}$ (inset: waveform of NBTI test) (d) temperature-dependent transfer
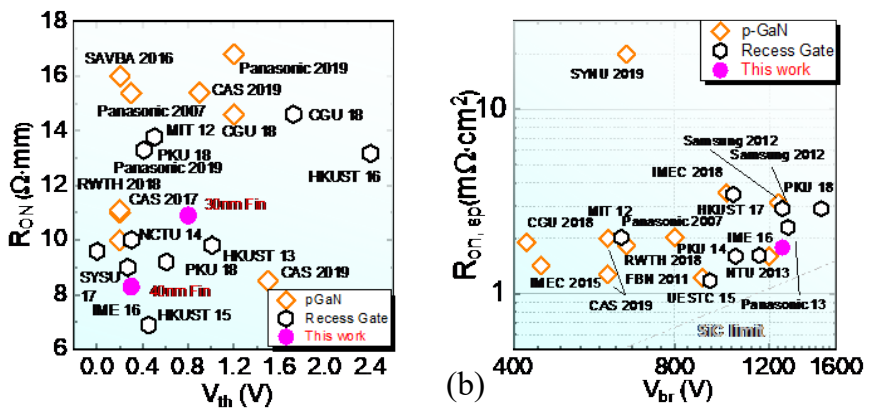

Figure 6. Benchmarking of (a) $R_{\text {on }}$ versus $V_{\text {th }}$ (defined at $1 \mu \mathrm{A} / \mathrm{mm}$ ) compared with e-mode GaN-on-Si HEMTs with $\mathrm{p}-\mathrm{GaN}$ gate and gate recess technology, (b) $R_{o n \text {, sp }}$ versus $V_{\text {br }}$ by defining $V_{\text {br }}$ at $I_{\text {off }} \leq 10 \mu \mathrm{A} / \mathrm{mm}$, for recess gate, floating substrate result are shown for a fair comparison. $\mathrm{R}_{\mathrm{on}, \mathrm{sp}}$ was calculated considering a $1.5 \mu \mathrm{m}$ of transfer length for each ohmic contact, which was taken into account for $\mathrm{R}_{\mathrm{on}, \mathrm{sp}}$ calculation. The $\mathrm{V}_{\mathrm{br}}$ results in the literature were recalculated by $\mathrm{I}_{\mathrm{off}} \leq 10 \mu \mathrm{A} / \mathrm{mm}$. Table. 1, comparison of reported e-mode TriGate AlGaN/GaN HEMTs.

Thanks to the improved electric field uniformity by the Tri-Gate structure, the devices could hold off-state voltages of $1270 \mathrm{~V}$ at $\mathrm{I}_{\text {off }}<10 \mu \mathrm{A} / \mathrm{mm}$ (under floating substrate) (Fig. 5 (a)). The forward gate current was compared for different gate metals and fin widths showing that $\mathrm{LiNiO}$ can works as a low leakage gate dielectric ( $\mathrm{Pd}$ gate was used in this work to mimic hole injection operation mode [2]) (Fig. 5 (b)). By replace the ohmic gate of Pd to Schottky gate Ti the positive gate leakage greatly reduced,
Table 1. Comparison of reported high performance e-mode tri-gate $\mathrm{AlGaN} / \mathrm{GaN}$ HEMTs

\begin{tabular}{cccccc}
\hline \hline References & This work & {$[4]$} & {$[5]$} & {$[6]$} & {$[3]$} \\
\hline Technologies & LiNiO & $\mathrm{NiO}_{\mathbf{x}}$ & Pt Gate & $\begin{array}{c}\text { Neg. } \\
\text { Charge }\end{array}$ & e-trap \\
$\mathbf{V}_{\mathbf{T H}}{ }^{\mathrm{a}}(\mathbf{V})$ & 0.5 & 0.45 & 0.6 & 0 & 2.61 \\
$\boldsymbol{S S} \mathbf{( m V / d e c})$. & 64 & 63 & 110 & 64 & 64 \\
$\mathbf{B V}(\mathbf{V})$ & 1200 & 650 & 1100 & 150 & 900 \\
$\mathbf{R}_{\mathbf{0 n}}(\mathbf{\Omega} \cdot \mathbf{m m})$ & 8.4 & 9.42 & 7.4 & 4.5 & 12 \\
$\mathbf{I}_{\mathbf{m a x}}(\mathbf{A} / \mathbf{m m})$ & 0.385 & 0.185 & 0.58 & 0.67 & 0.9 \\
$\mathbf{W}_{\text {Fin }}(\mathbf{n m})$ & 40 & 60 & 20 & 130 & 100 \\
\hline \hline
\end{tabular}

${ }^{\mathrm{a}}$ Extracted at $\mathrm{I}_{\mathrm{D}}=1 \mu \mathrm{A} / \mathrm{mm}$.

${ }^{b}$ Under floating substrate except third work, BV was taken at $\mathrm{I}_{\mathrm{D}}=10 \mu \mathrm{A} / \mathrm{mm}$.

${ }^{\mathrm{c}}$ Interpolating from fin width versus $\mathrm{V}_{\mathrm{TH}}$, the maximum fin width of e-mode operation (at $1 \mu \mathrm{A} / \mathrm{mm}$ ).

it proves the $\mathrm{LiNiO}$ is a good gate dielectric with low leakage. Positive gate leakage on different fin widths with ohmic gate was also studied, gate leakage is smaller under larger fin width device, step coverage difference of LiNiO film among different fin width devices and different flat-band voltage of side wall junction among different fin width devices could be the two reasons for this phenomenon. To evaluate the time-dependent $\mathrm{V}_{\text {th }}$ stability, NBTI test were done at $\mathrm{V}_{\mathrm{g}}=-30 \mathrm{~V}$, varying the temperature from $25^{\circ} \mathrm{C}$ to $150^{\circ} \mathrm{C}$. Small $\Delta \mathrm{V}_{\text {th }}$ of $-50 \mathrm{mV}\left(25^{\circ} \mathrm{C}\right)$, and $-100 \mathrm{mV}\left(150^{\circ} \mathrm{C}\right)$ respectively were observed under NBTI (Fig. 5 (c)). High temperature transfer characteristic revealed great high temperature $\mathrm{V}_{\text {th }}$ stability over other solutions using gate dielectrics to achieve e-mode operation[3].

Fig. 6 (a) benchmarks the $\mathrm{R}_{\text {on }}$ versus $\mathrm{V}_{\text {th }}$ among reported $\mathrm{p}-\mathrm{GaN}$ devices and gate recess MOSHEMTs, a small $R_{\text {on }}$ was achieved along with positive $\mathrm{V}_{\text {th }}$. The high-power figure-of-merit of the proposed device benchmarks very well against recess gate and p-GaN gate devices in the literature (Fig. 6(b)). Table 1 summarizes the device performance in this work and compares with other e-mode Tri-Gate devices. The device in this work presented a positive $\mathrm{V}_{\text {th }}$, achieved at a low $\mathrm{R}_{\text {sheet }}(275 \Omega / \mathrm{sq}$.) substrate. A good $R_{\mathrm{ON}}$ and $\mathrm{V}_{\mathrm{BR}}$ were shown at the same time. Tri-Gates require small fin widths to achieve e-mode operation, but by combining with $\mathrm{LiNiO}$, this requirement was significantly reduced, which led to a much smaller Ron for the same $\mathrm{V}_{\text {th. }}$.

\section{CONCLUSION}

These results show the enormous potential of $\mathrm{LiNiO}$ gate dielectric for high-performance and high-reliability e-mode GaN MOSHEMTs.

\section{REFERENCES}

[1] S. Kaneko et al., "Current-collapse-free operations up to $850 \mathrm{~V}$ by GaN-GIT utilizing hole injection from drain," in 2015 IEEE 27th International Symposium on Power Semiconductor Devices \& IC's (ISPSD), 10-14 May 2015 2015, pp. 41-44, doi: 10.1109/ISPSD.2015.7123384.

(C) 2021 IEEE. Personal use of this material is permitted. Permission from IEEE must be obtained for all other uses, in any current or future media, including reprinting/republishing this material for advertising or promotional purposes, creating new collective works, for resale or redistribution to servers or lists, or reuse of any copyrighted component of this work in other works. 
[2] Y. Uemoto et al., "Gate Injection Transistor (GIT) - A Normally-Off AlGaN/GaN Power Transistor Using Conductivity Modulation," IEEE Trans. Electron Devices, vol. 54, no. 12, pp. 3393-3399, Dec. 2007, doi: 10.1109/TED.2007.908601.

[3] C.-H. Wu et al., "Normally-Off Tri-Gate GaN MISHEMTs with $0.76 \mathrm{~m} \Omega \cdot \mathrm{cm} 2$ Specific On-Resistance for Power Device Applications," IEEE Trans. Electron Devices, vol. 66, no. 8, pp. 3441-3446, 2019, doi: 10.1109/ted.2019.2922301.

[4] Y. Ma et al., "Tri-gate GaN junction HEMT," Applied Physics Letters, vol. 117, no. 14, 2020, doi: $10.1063 / 5.0025351$.

[5] L. Nela, M. Zhu, J. Ma, and E. Matioli, "HighPerformance Nanowire-Based E-Mode Power GaN MOSHEMTs With Large Work-Function Gate Metal," IEEE Electron Device Lett., vol. 40, no. 3, pp. 439-442, 2019, doi: 10.1109/led.2019.2896359.

[6] H. Zhou, X. Lou, S. B. Kim, K. D. Chabak, R. G. Gordon, and P. D. Ye, "Enhancement-Mode AlGaN/GaN Fin-MOSHEMTs on Si Substrate With Atomic Layer Epitaxy $\mathrm{MgCaO}, "$ IEEE Electron Device Lett., vol. 38, no. 9, pp. 1294-1297, Sep. 2017, doi: 10.1109/LED.2017.2731993.

(C) 2021 IEEE. Personal use of this material is permitted. Permission from IEEE must be obtained for all other uses, in any current or future media, including reprinting/republishing this material for advertising or promotional purposes, creating new collective works, for resale or redistribution to servers or lists, or reuse of any copyrighted component of this work in other works. 